\title{
Superresolving masks for incoherent scanning microscopy
}

\author{
J. Grochmalicki,* E. R. Pike, and J. G. Walker \\ Department of Physics, King's College, Strand, London WC2R 2LS, UK
}

M. Bertero, P. Boccacci, and R. E. Davies

Dipartimento di Fisica del' Università di Genova, Via Dodecaneso 33, I-16146, Genova, Italy

\begin{abstract}
Received July 21, 1992; accepted September 28, 1992; revised manuscript received October 28, 1992
An approach to achieve superresolution in confocal scanning microscopy by using the singular-system approach to the inverse problem was recently proposed. It consists of using a specially designed mask that performs the task of data inversion by means of all-optical processing. We discuss an approximate binary form of such a mask that permits its practical manufacture for use in incoherent confocal microscopy. The performance characteristics of such an approximate mask are compared with those of an exact mask and with those of a conventional confocal scanning microscope. Although the resolution of the approximate microscope is slightly inferior to the exact one, they are both still advances over the conventional confocal one (the improvement of resolution being $65 \%$ and $70 \%$, respectively).
\end{abstract}

\section{INTRODUCTION}

A method of achieving superresolution in scanning microscopy by using specially designed optical masks was suggested recently ${ }^{1-3}$ and discussed in detail. This proposal is a refinement of an earlier attempt ${ }^{4-6}$ to apply the singular-system theory to the problem of data inversion in optical microscopy. In place of the single pinhole and detector of a confocal arrangement, the earlier design involved an array of detectors in the image plane. At each step of the scanning process their readouts were treated by a special inversion algorithm that was derived from singular-system analysis and that recovered the image at the point on axis. By means of scanning, a whole image would be formed. This image would be superresolving, i.e., the resolving power of such a microscope would be significantly improved compared with the standard confocal one. In Ref. 1 it was shown that the detector array and subsequent computer data processing can be replaced by a suitable optical mask located at the image plane that would perform the task of recovering the axial point by all-optical means. The design of the mask is also based on singular-system theory (it implements essentially the same inversion algorithm as before), and the resultant image is strictly equivalent to the previous one, i.e., it is also superresolving. For the case of a coherent confocal microscope, this has been verified experimentally ${ }^{3}$ (in both one and two dimensions).

In the case of a microscope that uses incoherent light, the practical realization of the theoretically calculated mask with a continuously changing profile proves to be prohibitively difficult. Hence the need arises for a modified mask design that not only preserves as much of the resolving power of the microscope as possible but also is easy to manufacture. In this paper we present such a design, developed for the sake of practical utilization in experiment (such an experiment is currently under way). We focus here on the case of two circular pupils and, to facilitate numerical computation, a small numerical aper- ture. The method itself, however, is general and can be applied equally well for any numerical aperture.

The paper is organized as follows. In Section 2 we review briefly the singular-system approach to data inversion with particular emphasis on the concept of the optical mask. In Section 3 we describe the new method of practical mask implementation to be used in the superresolving incoherent microscope. Finally, in Section 4 we analyze the imaging characteristics of the true mask, i.e., the mask that was theoretically calculated, with the new approximate one. Although the resolving power of a microscope that uses the new mask deteriorates slightly, it still proves to be far superior to that of a conventional confocal microscope.

\section{SINGULAR-SYSTEM THEORY OF THE OPTICAL MASK}

In the singular-system approach to data inversion in scanning microscopy, one solves the Fredholm equation of the first kind, which describes the imaging system, by finding its singular-value spectrum and its object and image singular functions. These functions provide the complete orthonormal basis sets for the object and image. One then performs the inversion by finding the coefficients of the singular-function expansion up to a point (the truncation index $K$ ) that is determined by the level of noise in the image and the singular-value spectrum.

The imaging relationship between the object $f$ and the image $g$ for a given scanning position can be written as

$$
g(x)=(A f)(x)=\int S_{2}(x-y) S_{1}(y) f(y) \mathrm{d} y,
$$

where $S_{1}$ and $S_{2}$ represent the point-spread functions (PSF's) of the illuminating and imaging lenses, respectively. $x$ and $y$ are the two-dimensional variables in the image and object planes, respectively. In the incoherent case both $f$ and $g$ are intensity distributions. 
The inverse problem is that, having measured $g(x)$, we seek to recover $f(y)$ or, because scanning is involved, only $f(0)$, the object value at the axial point. Complete reconstruction of $f$ is then achieved after we solve this problem at each scanning position. Our approach here is based on the singular-system analysis. The singular system $\left\{\alpha_{k} ; u_{k}, v_{k}\right\}$, where $k=1,2, \ldots$ associated with the operator $A$ [cf. Eq. (2.1)], is defined by

$$
A u_{k}=\alpha_{k} v_{k}, \quad A^{*} v_{k}=\alpha_{k} u_{k}
$$

with $A^{*}$ denoting the adjoint operator of $A$ :

$$
\left(A^{*} g\right)(y)=S_{1}(y) \int S_{2}(x-y) g(x) \mathrm{d} x .
$$

The singular functions $u_{k}$ and $v_{k}$ are basis functions for representing object and image, respectively, whereas $\alpha_{k}$ denotes the $k$ th singular value of the system. In these terms we can express the best approximation of $f(0)$ as

$$
\tilde{f}(0)=\sum_{k=1}^{K} \frac{\left(g, v_{k}\right)}{\alpha_{k}} u_{k}(0) .
$$

As is mentioned above, the truncation index $K$ is controlled by the level of noise that affects the data. ${ }^{4}$ The parentheses above denote the standard function space scalar product, i.e.,

$$
\left(g, v_{k}\right)=\int g(x) v_{k}(x) \mathrm{d} x
$$

If we now write

$$
M(x)=\sum_{k=1}^{K} \frac{1}{\alpha_{k}} u_{k}(0) v_{k}(x),
$$

we obtain the previous expression for $\tilde{f}(0)$ in a different form:

$$
\tilde{f}(0)=\int g(x) M(x) \mathrm{d} x=(g, M) .
$$

Thus the implementation of the inversion algorithm involves only the operation of multiplying the image $g(x)$ by the known function $M(x)$ and then the spatial integration of the product. Both these operations can be implemented optically to enable implementation of the data inversion before detection. One can achieve this by placing in the image plane an optical mask with a transmission profile given by $M(x)$ and then using a spatially integrating detector, or detectors, which measures directly the recovered axial object point $\tilde{f}(0)$. The details of possible optical arrangements are discussed in Ref. 1.

In this type of arrangement the spatial distribution $g(x)$ is not being measured; therefore detection noise cannot affect the inversion. However, the optical mask itself will never be perfect, i.e., described exactly by the function $M(x)$, because of both fabrication and implementation difficulties. Therefore the mask imperfections will assume the role of the noise in the system. If we note that $g$ and $M$ enter the inversion formula [Eq. (2.7)] in precisely the same manner, it becomes evident that the argument regarding the truncation of the singular-function expansion holds here unchanged. In place of noise affecting the image, we must now consider the deviation of the actual mask from the ideal one, as defined by the analytical expression [Eq. (2.6)], with $K \rightarrow \infty$. This means that if our mask-fabrication technique or the mask-alignment procedure is to a certain degree inaccurate, using a mask in which the truncation index $K$ is too large will actually propagate this error to the readouts. For this reason the difficult task of manufacturing the complicated masks that correspond to some large values of $K$ makes little sense because they are much more fragile than the low- $K$ masks and more sensitive to the practical inaccuracies. In fact, it seems much better to use a mask with $K$ that is too low, although in this case the resolving power will inevitably deteriorate. In Section 3 we use a value of $K=5$.

\section{NOVEL MASK DESIGN}

In the present paper we are interested in the special case of a scanning microscope that has circular pupils, is of low numerical aperture, and is illuminated by incoherent light. For this case the PSF's $S_{1}$ and $S_{2}$ are equal:

$$
S_{1}(x)=S_{2}(x)=\left[\frac{2 J_{1}(\pi \rho)}{\pi \rho}\right]^{2}, \quad \rho=|x| .
$$

The singular system for this case can only be determined numerically. This is done by discretizing the operator $A$ by using sampling theory ${ }^{7}$ and then by performing numerical computation of the singular system. With the use of these components one can evaluate the mask function. Figure 1 shows the form of the mask function for $K=5$. It has a continuous profile and changes sign. These features unfortunately make it rather difficult to implement the mask for the case of incoherent light because it is not possible to have a negative light intensity. However, if two detectors are used (with separate light processing) and the final readout is formed by subtraction of their signals, one can obtain easily the negative result. ${ }^{1}$ The practical difficulty, however, of how to implement the continuous profile accurately remains.

The solution we propose here is to use a partially reflecting and partially transparent plate inserted at $45^{\circ}$ into the light beam in the image plane (see Fig. 2). The light that

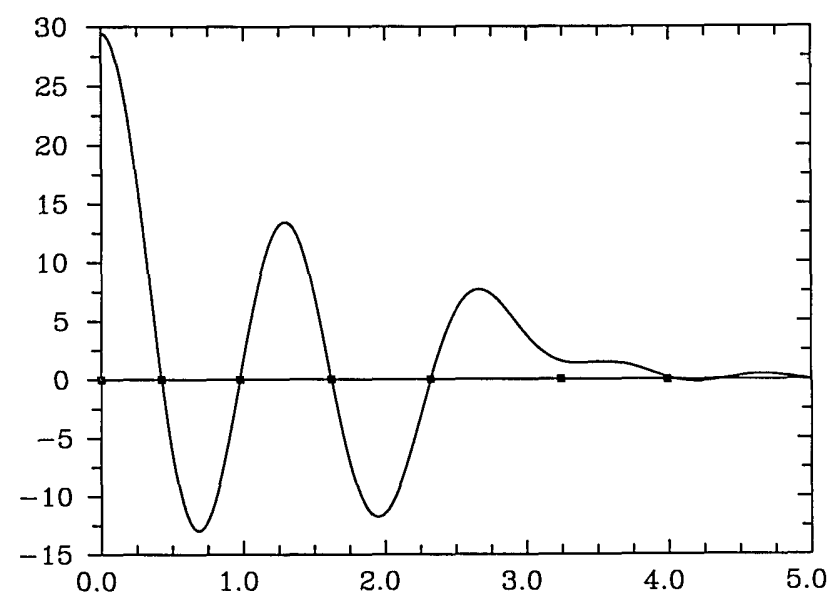

Fig. 1. Arbitrarily normalized image-plane mask $M(\rho)$ for incoherent imaging that we constructed from the first five singular functions. 


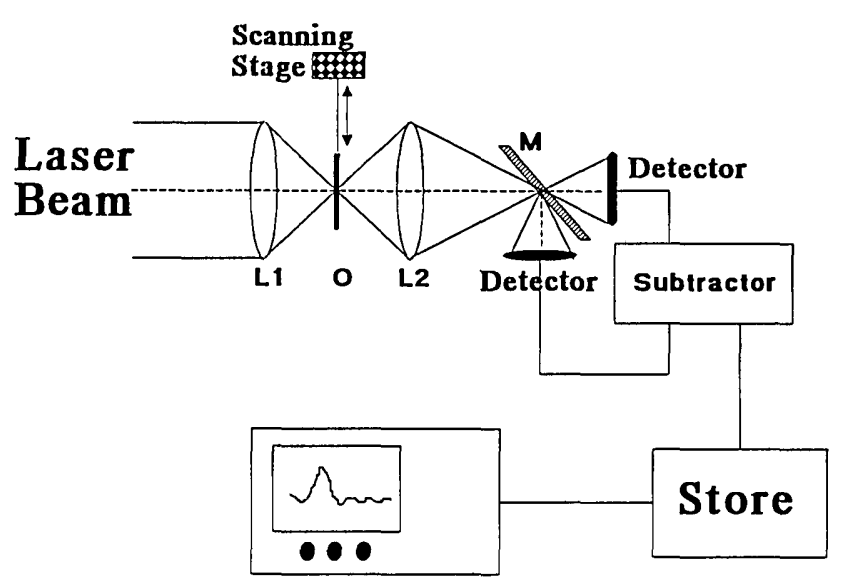

Fig. 2. Incoherent confocal microscope setup with the elliptical annular mask that we inserted at $45^{\circ}$ in the image plane: L's, lenses; $M$, masks.

passes through the uncoated parts of such a mask (and measured by one detector) corresponds to the positive components of the mask profile, whereas the light that is reflected at $90^{\circ}$ (and measured by a separate detector) corresponds to the negative components. The mirror design consists of a set of prolate elliptical annuli (forming circles when orthoprojected onto the image plane). Their positions and widths are chosen to emulate as closely as possible the action of the actual mask. The basic limitation is to have no annuli that are too thin.

The precise algorithm we chose is as follows. First, for the case of $K=5$, we take the first five zeros of $M(x)$ and supplement them with one additional point between the fourth and the fifth zeros (because this interval is approximately two times longer than the rest), as may be seen in Fig. 1. Each of the six intervals formed by these points is then divided into three equal subintervals, and the radial weight covered by the profile of $M(x)$ over each of these 18 subintervals is calculated. Next each subinterval is replaced with a centrosymmetric step pattern $(+1,-1,+1)$, where $M(x)$ is positive valued, or $(-1,+1,-1)$, where it is negative, with the step positions chosen so that the radial weight is equal to the radial weight of $M(x)$ within that subinterval.

For the first three subintervals the algorithm is slightly modified. The first subinterval defines the actual scaling; hence a constant value is used throughout this subinterval. Using the $(+1,-1,+1)$ pattern for the next two subintervals results in extremely thin annuli (the -1 parts). Thus in these subintervals we used a $(+1,-1)$ pattern in the second subinterval and $a(-1,+1)$ in the third to overcome this difficulty.

As a result of the above prescription the radial weight is reproduced within each subinterval, and the original zero crossings of the continuous profile $M(x)$ are also preserved. The mask that we constructed according to the above prescription $M_{a}(x)$ is shown in Fig. 3 .

In mathematical terms, an attempt is being made to replace the continuous profile $M(x)$ by a multiple-step function (with two values +1 and -1 ), which is, in a sense, close to the original in the function space. Obviously our choice of a function-space measure is quite arbitrary, the only justification being that satisfactory performance is achieved, as shown in Section 4 below.

\section{PERFORMANCE ANALYSIS}

To estimate the performance characteristics of the new optical processor by using the approximate mask $M_{a}(x)$, we calculate its instrumental impulse-response function or effective PSF. By modifying the inversion formula [Eq. (2.7)] with the general mask $m(x)$ and remembering that $g=A f$, we have

$$
\tilde{f}(0)=(m, g)=(m, A f)=\left(A^{*} m, f\right),
$$

or, equivalently, if we let $T=A^{*} m$, we obtain

$$
\tilde{f}(0)=\int T(y) f(y) \mathrm{d} y .
$$

After we include the effect of the scanning translations, Eq. (4.2) yields an expression for the recovered image:

$$
\tilde{f}(z)=\int T(y) f(z-y) \mathrm{d} y=(T * f)(z) .
$$

The function $T(y)$ thus describes the impulse-response function that is required. In explicit terms,

$$
T(y)=S_{1}(y) \int S_{2}(x-y) m(x) \mathrm{d} x .
$$

The Fourier transform $\hat{T}(\omega)$ describes the frequency response or transfer function of the microscope.

To investigate the actual performance of the microscope, we evaluate both these characteristics for the two calculated masks, the continuous $M(x)$ and the binary $M_{a}(x)$. We also compare them with the corresponding characteristics of a conventional incoherent confocal scanning microscope for which the PSF $t(y)$ is given by Ref. 8:

$$
t(y)=\left[\frac{2 J_{1}(\pi \rho)}{\pi \rho}\right]^{4}, \quad \rho=|x| .
$$

All relevant calculations are performed numerically by means of a novel precise algorithm for the Hankel transform (the axially symmetrical Fourier transform). The resulting PSF's $T(y)$ (corresponding to the continuous mask) and $T_{a}(y)$ (corresponding to the binary mask) are compared with $t(y)$ in Fig. 4. It may be seen that both the mask microscope PSF's are considerably sharper than

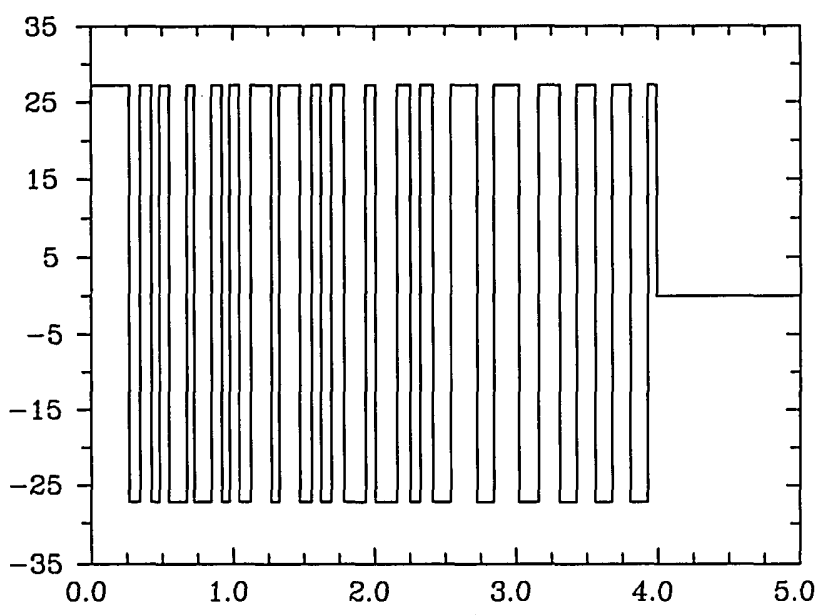

Fig. 3. Approximate binary mask design $M_{a}(\rho)$ corresponding to the exact mask that is shown in Fig. 1. 


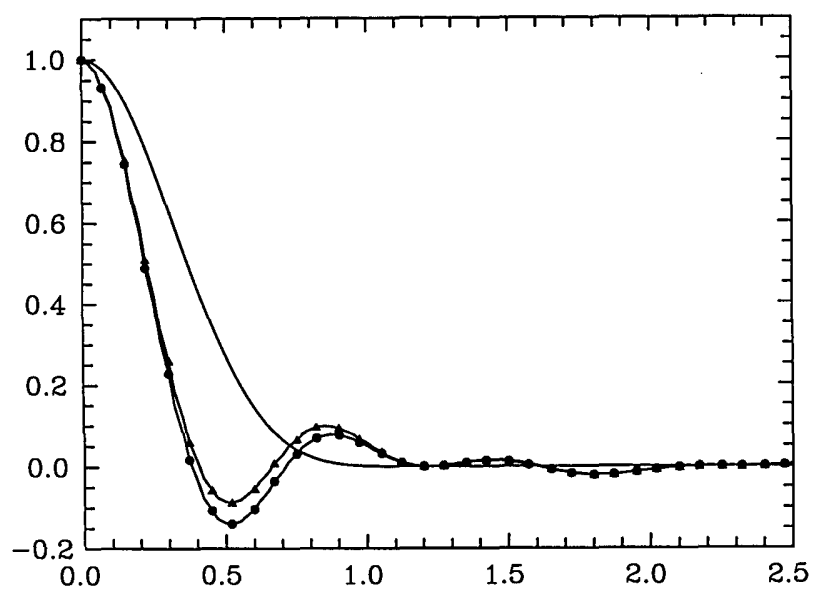

Fig. 4. PSF's of the exact mask $T(\rho)$ (circles) and approximate binary mask $T_{\alpha}(\rho)$ (triangles) compared with the conventional confocal microscope $t(\rho)$ (solid curve). Both masks show superresolution inasmuch as their profiles are significantly narrower than that of the confocal microscope.

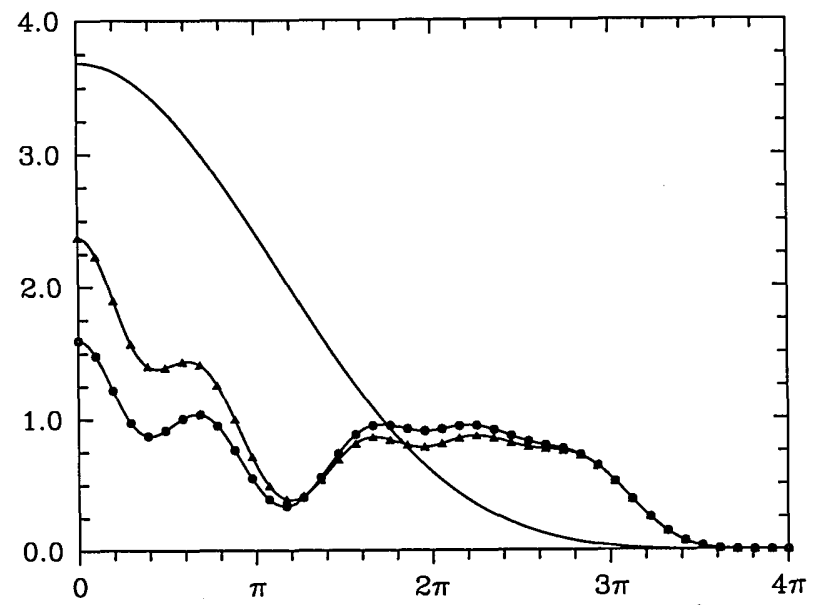

Fig. 5. Transfer functions of the exact mask $\hat{T}(\omega) \overrightarrow{\text { (circles) and }}$ approximate mask $\hat{T}_{a}(\omega)$ (triangles) compared with the confocal microscope $\hat{t}(\omega)$ (solid curve). Both masks display considerably stronger frequency response in the upper half of the spectrum.

that of the confocal design and could be described as superresolving. As one might reasonably expect, the PSF for the binary mask is slightly broader than that for a continuous mask. If we adopt, according to Ref. 8, the width of the central peak at its half-maximum as a measure of the resolution, the continuous mask achieves a $70 \%$ increase in resolution over a conventional confocal scanning microscope (ratio of half-height widths is 1.7).
The approximate binary mask design achieves an increase of $65 \%$.

The corresponding volume-normalized transfer functions are displayed in Fig. 5. The PSF's $T(y)$ and $T_{a}(y)$ are band limited with the same band as the conventional confocal microscope. The improvement in resolution can be seen in the behavior of $\hat{T}(\omega)$ and $\hat{T}_{a}(\omega)$ in the highfrequency region of the spectrum. The frequency responses of both the superresolving microscopes are considerably stronger in this region and are more similar to the ideal flat profile.

The above results confirm the superresolving characteristics of an approximate binary mask design and indirectly sustain the heuristically adopted mask-design method. An experimental mask has been fabricated according to this prescription and is currently being tested.

\section{ACKNOWLEDGMENTS}

R. E. Davies is grateful for a Royal Society Overseas Fellowship, and J. Grochmalicki, E. R. Pike, and J. G. Walker acknowledge the support of the U.K. Science and Engineering Research Council.

*On leave from Institute for Theoretical Physics, Polish Academy of Sciences, Aleja Lotników 32/46, 02-668 Warsaw, Poland.

\section{REFERENCES}

1. J. G. Walker, E. R. Pike, R. E. Davies, M. R. Young, G. J. Brakenhoff, and M. Bertero, "Superresolving scanning optical microscopy using holographic optical processing," J. Opt. Soc. Am. A 1, 59-64 (1993).

2. M. Bertero, P. Boccacci, R. E. Davies, F. Malfanti, E. R. Pike, and J. G. Walker, "Superresolution in confocal scanning microscopy: IV. Theory of data inversion by the use of optical masks." Inverse Probl. 8, 1-23 (1992).

3. M. R. Young, S. H. Jiang, R. E. Davies, J. G. Walker, E. R. Pike, and M. Bertero, "Experimental confirmation of superresolution in coherent confocal scanning microscopy using optical masks," J. Microsc. 165, 131-138 (1992).

4. M. Bertero, P. Boccacci, M. Defrise, C. De Mol, and E. R. Pike, "Superresolution in confocal scanning microscopy: II. The incoherent case," Inverse Probl. 5, 441-461 (1989).

5. M. Bertero, P. Boccacci, R. E. Davies, and E. R. Pike, "Superresolution in confocal scanning microscopy: III. The case of circular pupils," Inverse Probl. 7, 655-674 (1991).

6. E. R. Pike, "Inverse problems in confocal microscopy," in Inverse Problems in Scattering and Imaging, M. Bertero and E. R. Pike, eds. (Hilger, Bristol, 1992), pp. 164-179.

7. M. Bertero and P. Boccacci, "Computation of the singular system for a class of integral operators related to data inversion in confocal microscopy," Inverse Probl. 5, 935-957 (1989).

8. T. Wilson and C. Sheppard, Theory and Practice of Scanning Optical Microscopy (Academic, London, 1984). 